and QCA with respect to percentage of stenosis, minimum luminal diameter was $(2.04 \pm 0.18) \mathrm{mm}$ vs $(2.0 \pm 0.17) \mathrm{mm}$, respectively, the correlation index $\mathrm{R}=0.627$. Reference segment diameter was $(3.28 \pm 0.19) \mathrm{mm}$ vs $(3.17 \pm 0.21) \mathrm{mm}$, respectively, the correlation index $\mathrm{R}=0.782$. In contrast, there was a difference in the assessment luminal diameter stenosis with minimum luminal area. The stenosis detected by IVUS could find the severity of stenosis, could reduce much MACE relatively.

Conclusions In patients with angiographically intermediate lesions, the frequency of severe stenosis detected by IVUS were high, indicating that angiography underestimated the severity of stenosis. Smoking and Hypertension could be used to stratify these lesions into groups with higher risk of MACE.

\section{e0535 THE ABILITY OF OPTICAL COHERENCE TOMOGRAPHY FOR ASSESSMENT OF INTERMEDIATE CORONARY STENOSIS COMPARISON WITH OUANTITATIVE CORONARY ANGIOGRAPHY}

doi:10.1136/hrt.2010.208967.535

Fang Zhe, Zhou Yujie, Shi Dongmei, Zhao Yingxin, Liu Yuyang, Li Yanfang. Department of Cardiology, Beijing Anzhen Hospital, Capital Medical University, Beijing, China

Objective Optical coherence tomography is a new intravascular imaging method with a high resolution of approximately $10 \mu \mathrm{m}$. This study aims to examine quantitative optical coherence tomography (OCT) derived measurements intermediate coronary stenosis from quantitative coronary angiography (OCA).

Methods 240 patients with coronary intermediate stenosis by quantitative coronary angiography (OCA) and underwent OCT assessment of the lesions artery. The results from OCA and OCT were compared using unpaired t-test. Multiple regression analysis was performed. The latter of MACE was significantly lower than that by QCA detected.

Results A total of 118 stenotic coronary lesions were classified as intermediate by OCA. Subgroup of the plaque was analysed, OCT could estimate the fibrous cap thickness, and it was $122 \pm 24.7 \mu \mathrm{m}$ ), the reference segment diameter was $3.06 \pm 0.12 \mathrm{~mm}$ ), the minimum luminal diameter was $1.94 \pm 0.12 \mathrm{~mm}$ ). The minimum luminal area was $4.8 \pm 1.17 \mathrm{~mm}^{2}$ ). At 12 months of clinical follow-up, 8 patients was found to have MACE. It was much lower than QCA group.

Conclusions In patients with angiographically intermediate lesions, the frequency of severe stenosis detected by OCT were high, indicating that angiography underestimated the severity of stenosis. This unique resolution of OCT suggests that it may be well suited for identifying vulnerable plaques in patients at risk. Optical coherence tomography is a feasible imaging modality in patients and allows us to identify quantitative plaque characters, such as fibrous cap, vulnerable plaque.

\section{E0536 COMPARATIVE EFFECTS OF PERCUTANEOUS CORONARY INTERVENTION FOR CULPRIT CORONARY ARTERY OR TOGETHER WITH NON-CULPRIT CORONARY ARTERY IN PATIENTS WITH MULTI-VESSEL CORONARY ARTERY DISEASE}

doi:10.1136/hrt.2010.208967.536

Yu Miao, Zhou Yujie, Wang Zhijian, Shi Dongmei, Liu Yuyang, Zhao Yingxin, Guo Yonghe. Anzhen Hospital Capital Medical University

Background Rapid recanalisation of the culprit lesion is the main goal of primary angioplasty for acute ST-segment elevation myocardial infarction (STEMI), but strategy for treatment of culprit and non-culprit lesions in multi-vessel coronary artery disease remains unclear. Objectives Aims to examine the 6-month outcomes for non-culprit interventions performed at the time of the primary percutaneous coronary intervention (PCI) in multi-vessel coronary artery disease.

Methods A total of 1120 patients treated with primary angioplasty between 2008 and 2009 were classified in groups of patients with multi-vessel coronary artery disease (MVD). We examined the associated 6 month outcomes following non-culprit interventions performed at the time of primary PCI. Patients were subdivided in two groups on the basis of the revascularization strategy: 1) patients undergoing PCI of the culprit coronary artery only; 2) patients undergoing PCI of both the culprit coronary artery and non-culprit coronary artery during the initial procedure. All the patients were followed up for 6-month for major adverse cardiac events (MACE). Results The two groups did not differ with respect to baseline clinical and angiographic characteristics. At 6-month, compared with PCI restricted to the culprit coronary artery only, multivessel PCI was associated with higher rates of re-infarction $(7.9 \%$ vs $2.6 \%$, $\mathrm{p}<0.001)$, revascularization $(14.5 \%$ vs $6.8 \%, \mathrm{p}<0.001)$, and MACEs $(26.7 \%$ vs $14.8 \%, \mathrm{p}<0.001)$.

Conclusions Non-culprit coronary interventions were significantly associated with increased mortality. Our data suggest that in patients with MVD, primary PCI should be directed at the culprit coronary artery only, with decisions about PCI of non-culprit lesions guided by objective evidence of residual ischaemia at late follow-up. Further studies are needed to confirm these findings.

\section{e0537 OCCLUDER DISLOCATION - THE SEVERE COMPLICATION OF TRANSCATHETER CLOSURE OF PATENT DUCTUS ARTERIOSUS WITH PULMONARY ARTERIAL HYPERTENSION}

doi:10.1136/hrt.2010.208967.537

Liu Zhenjiang, Shen Xiangqian, Fang Zhenfei. Department of Cardiology, The Second Xiangya Hospital, Central South University

Patent ductus arterious (PDA) is common congenital heart disease in children and infancy, closure of PDA by surgery or interventional cardiac catheterisation is safe now, but those with large and untreated patent ductus arteriosus usually developed pulmonary hypertension in adults, in which the surgery to ligament PDA become a challenge because the high risk of the complication. Recent years some authors have reported that transcatheter closure of patent ductus arteriosus with pulmonary arterial hypertension successfully. ${ }^{12}$ Our group had treated 284 cases of PDA patients by transcatheter since 2001, which included 25 cases with pulmonary arterial hypertension, among them the PDA of 19 cases were closured successfully, 4 cases were abandoned because of the device was not available or the symptoms became worsening in the catheter room, in the other 2 cases the occluder fell of $48 \mathrm{~h}$ after operation and caused serious complication, here we reported it.

\section{C0538 THANSCATHETER THERAPY OF GIANT VENTRICULAR SEPTAL DEFECT ACCOMPANIED WITH CRITICAL PULMONARY VALVE STENOSIS AND HIGH DEGREE A-V BLOCK: A CASE REPORT WITH 4 YEAR FOLLOW-UP}

doi:10.1136/hrt.2010.208967.538

Zhongru Ding, Yongwen Qin. Department of Cardiology, The 94th Military Hospital, Nanchang, Jiangxi Province, Pr China

Transcatheter closure of ventricular septal defects, which has been used extensively in recent years, has become a treatment of choice in selected patients. However, there is relatively limited experience with large VSD (>15 mm) and/or obviously preprocedural ECG 